\title{
State Reconstruction in Spatially Distributed BioProcess Systems using Reduced Order Models: Application to the Gluconic Acid Production.
}

\author{
Míriam R. García, Carlos Vilas, Julio R. Banga, Velislava N. Lyubenova, Maya \\ N. Ignatova, Antonio A. Alonso
}

\begin{abstract}
In this work, the dissipative nature of spatially distributed bioprocess systems is exploited to develop efficient state observers based on a low dimensional dynamic representation of the original set of partial differential equations. The approach we suggest combines standard observer design techniques for bioreactors with efficient model reduction methodologies based on projection of the original concentration fields on low dimensional subspaces capturing the slow dynamics of the process.

Aspects related with the location of sensors and their influence on the ability to reconstruct concentration fields will also be considered. Finally, the different aspects of the methodology, as well as the efficiency of the resulting observers will be illustrated on a case study of industrial interest, namely a tubular bioreactor producing gluconic acid by Aspergillus Niger.
\end{abstract}

\section{INTRODUCTION}

As pointed out by [1], on-line monitoring and control in biotechnology have been hampered by a number of obstacles essentially associated with the poor knowledge of the process and the lack of reliable sensors capable of providing online measurements of the biochemical variables. To overcome these limitations, a theoretical identification framework has been developed by [1] which exploits the underlying reaction structure and transfer mechanisms of the biotechnological processes to systematically design and implement state and parameter identification schemes. This theory has been extended to particular classes of distributed reactors and bioreactors (see for instance [2] and [3]). However, a number of issues and questions related with the spatially distributed nature of this class of systems still remain open.

In fact, on-line state observation is particularly critical in spatially distributed bio-reactors due to the high dimensionality associated with the dynamic representation. In this class of systems, the states have to be described from microscopic conservation laws for mass and energy balances which result into a nonlinear set of partial differential equations. The solution of these systems usually involves the integration of a large set of ordinary differential equations [4]. In addition the observer must be supplied with on-line state measurements covering the whole spatial domain which are usually unavailable due to the limited number of sensors [2] [5] [6].

M. R. García, C. Vilas, J. R. Banga and A. A. Alonso are with the Process Engineering Group, Instituto de Investigaciones Marinas (CSIC) C/Eduardo Cabello 6, 36208 Vigo, Spain antonio@iim.csic.es

V. N. Lyubenova and M. N. Ignatova are with the Institute of Control and System Research, Bulgarian Academy of Sciences, P.O. Box 79, 1113 Sofia Bulgaria
In order to overcome these issues, we exploit the dissipative nature of diffusion-convection reaction systems [7], [8] and adapt the results developed in [9] and [10] to derive exponential state observers based a low dimensional dynamic representation of the original system of partial differential equations. This framework will also be useful in devising a systematic solution to the field reconstruction problem from a limited number of measurements. To that purpose, we extend the formalism proposed by [6] to take advantage of the finite element method [11] and formulate a generalized version of the optimal sensor placement problem.

The approach presented in this work will be illustrated on a continuous tubular reactor for the production of gluconic acid. This process, of relevance in the food and pharmaceutical industry [12], has been largely studied by [13] which gives a detailed description of the reaction mechanism and therefore results convenient for validation purposes.

The paper is structured as follows: the design and error convergence analysis of distributed observers is presented in Section II. In Section III, the model reduction technique is described and applied to the design of low-dimensional observers and state reconstruction schemes from a reduced number of sensors. Continuous production of gluconic acid, described in section IV, will be the process employed to validate these techniques in section V. Finally, conclusions are summarized in section VI.

\section{OBSERVER DESIGN}

As pointed out by [1] observers in biochemical processes are required either because of the absence of knowledge of the reaction rates or the lack of cheap and reliable online sensors. In this section we present and adapt the theory developed by [1] and [3] on bioreactor observer design to spatially distributed tubular bioreactors and discuss their convergence properties.

\section{A. General dynamic representation of Biochemical tubular reactors}

Tubular bioreactors are diffusion-convection-reaction systems described by spatially distributed mass and energy balances which mathematically translates into a set of Partial Differential Equation (PDEs) with the following general dynamical structure:

$$
\frac{\partial x}{\partial t}=D \frac{\partial^{2} x}{\partial \xi^{2}}-v \frac{\partial x}{\partial \xi}+K \varphi(x)+Q\left(x^{*}-x\right)
$$

where $x(t, \xi) \in \mathbb{R}^{s}$ represents the state vector field as a function of time and the spatial coordinates $\xi \in \mathbb{R}^{h}(h=1$ 
in reactors with axial dispersion). $v \in \mathbb{R}$ denotes flow velocity, $D \in \mathbb{R}^{s \times s}$ is a matrix of dispersion coefficients and $Q \in \mathbb{R}^{s \times s}$ includes parameters describing mass and energy exchange with the environment, being $x^{*} \in \mathbb{R}^{s}$ its field reference. Matrices $D$ and $Q$ are diagonal and bounded by numbers $\delta>0$ and $q \geq 0$ as:

$$
\|D\| \geq \delta \quad\|Q\| \geq q
$$

In addition, the kinetic part is described by a vector of reaction rates $\varphi(x) \in \mathbb{R}^{r \times 1}$ and a full rank matrix $K \in$ $\mathbb{R}^{s \times r}$ of yield coefficients. Finally, system's description is completed with appropriate boundary and initial conditions, which for tubular reactors with axial dispersion are:

$$
\begin{gathered}
D \frac{\partial x}{\partial \xi}=-v\left(x_{i n}-x\right) \quad \forall t \in \mathbb{R}^{+}, \xi=0, \\
\frac{\partial x}{\partial \xi}=0 \quad \forall t \in \mathbb{R}^{+}, \xi=L \\
\text { and } x=x_{0} \quad \forall \xi \in[0, L], t=0
\end{gathered}
$$

where (3a) and (3b) are the so-called Danckwerts boundary conditions with $L$ being the longitudinal length of the reactor, $x_{i n}$ the input and $x_{0}$ the initial condition.

\section{B. State reconstruction without knowledge of the reactions} rates

The development of distributed state observers which do not require knowledge of the reaction rates relies on the principles of mass and energy conservation [14] which essentially state the existence of some unaltered entities collected on a vector $\widetilde{z}$ and related to the state vector $x$ by a linear map of the form $\widetilde{z}=B^{T} x$. Since these entities are not produced, neither destroyed by reaction, but only transported through the system, the columns of $B$ must form a basis for the null space of $K^{T}$ so that $B^{T} K=0$. In addition, since $K$ is full rank $(r)$, the dimension of the $K$ left null space is $s-r$. This fact allows us to define the following partitions in $K$ and $B$, respectively:

$$
B^{T}=\left[B_{e}^{T} \mid B_{m}^{T}\right] \quad K^{T}=\left[K_{e}^{T} \mid K_{m}^{T}\right]
$$

where $K_{m}^{T} \in \mathbb{R}^{(r \times r)}$ and $B_{e}^{T} \in \mathbb{R}^{(s-r \times s-r)}$ are invertible. In addition, since $B^{T} K=0$ we also have that:

$$
B_{m}^{T}=-B_{e}^{T} K_{e} K_{m}^{-1}
$$

On the other hand, $\widetilde{z}$ can be written as:

$$
\widetilde{z}=B^{T} x=B_{e}^{T} x_{e}+B_{m}^{T} x_{m}
$$

Substituting (4) into (5) and re-ordering terms, we get:

$$
\widetilde{z}=B_{e}^{T}\left(x_{e}-K_{e} K_{m}^{-1} x_{m}\right)
$$

Finally, by defining $z=B_{e}^{-T} \widetilde{z}$ a new transformation is obtained of the form:

$$
z=x_{e}+A_{o} x_{m} \quad \text { with } \quad A_{o}=-K_{e} K_{m}^{-1}
$$

This transformation, that coincides with the one proposed by [1] and [3], determines which states can be observed $\left(x_{e} \in \mathbb{R}^{(s-r)}\right)$ form measurements $x_{m} \in \mathbb{R}^{r}$, provided that a reliable estimation $z$ is at hand. As shown next, the evolution of $z$ is independent of the reaction rates. To that purpose, let us partition the original system (1) into the sets of observed and measured states as follows:

$$
\begin{aligned}
\frac{\partial x_{e}}{\partial t} & =D_{e} \frac{\partial^{2} x_{e}}{\partial \xi^{2}}-v \frac{\partial x_{e}}{\partial \xi}+K_{e} \varphi(x)+Q_{e}\left(x_{e}^{*}-x_{e}\right) \\
\frac{\partial x_{m}}{\partial t} & =D_{m} \frac{\partial^{2} x_{m}}{\partial \xi^{2}}-v \frac{\partial x_{m}}{\partial \xi}+K_{m} \varphi(x)+Q_{m}\left(x_{m}^{*}-x_{m}\right)
\end{aligned}
$$

with:

$D=\left[\begin{array}{cc}D_{e} & 0 \\ 0 & D_{m}\end{array}\right] \quad K=\left[\begin{array}{c}K_{e} \\ K_{m}\end{array}\right] \quad Q=\left[\begin{array}{cc}Q_{e} & 0 \\ 0 & Q_{m}\end{array}\right]$

Applying (7) to (8) we obtain the following reaction rate independent set of PDEs for the new states $z$ :

$$
\begin{gathered}
\frac{\partial z}{\partial t}=D_{e} \frac{\partial^{2} z}{\partial \xi^{2}}-v \frac{\partial z}{\partial \xi}+Q_{e}\left(z^{*}-z\right)+h\left(x_{m}\right) \\
h\left(x_{m}\right)=\left(A_{o} D_{m}-D_{e} A_{o}\right) \frac{\partial^{2} x_{m}}{\partial \xi^{2}}+ \\
+\left(A_{o} Q_{m}-Q_{e} A_{o}\right)\left(x_{m}^{*}-x_{m}\right)
\end{gathered}
$$

so that the observer structure becomes:

$$
\begin{gathered}
\frac{\partial \widehat{z}}{\partial t}=D_{e} \frac{\partial^{2} \widehat{z}}{\partial \xi^{2}}-v \frac{\partial \widehat{z}}{\partial \xi}+Q_{e}\left(z^{*}-\widehat{z}\right)+h\left(x_{m}\right) \\
\widehat{x}_{e}=\widehat{z}-A_{o} x_{m}
\end{gathered}
$$

The convergence properties of this observation scheme are summarized in the following proposition where integrals over the domain $\Omega$ and over the boundary domain $\Gamma$ are denoted by:

$$
\langle f, g\rangle_{\Omega}=\int_{\Omega} f g d \xi \quad \text { and } \quad\langle f, g\rangle_{\Gamma}=\int_{\Gamma} f g d \Gamma
$$

and the $L_{2}$ norm is defined as:

$$
\|f\|_{\Omega}^{2}=\langle f, f\rangle_{\Omega}
$$

\section{Proposition 1}

Let the evolution of $z$ and $\widehat{z}$ be described by (9) and (10), respectively. Then $\widehat{z}$ will converge exponentially to $z$ in the norm defined by (12).

Proof: First, we define the error between the real and the observed states as $e=x_{e}-\widehat{x}_{e}=z-\widehat{z}$ and construct the error evolution equation by combining (10) and (9) so that:

$$
\frac{\partial e}{\partial t}=D_{e} \frac{\partial^{2} e}{\partial \xi^{2}}-v \frac{\partial e}{\partial \xi}-Q_{e} e
$$

Now, let us define a Lyapunov function candidate $\mathcal{V}=$ $\frac{1}{2}\langle e, e\rangle_{\Omega}$ and compute its time derivative along (13) so that:

$$
\frac{d \mathcal{V}}{d t}=\left\langle e, D_{e} \frac{\partial^{2} e}{\partial \xi^{2}}\right\rangle_{\Omega}-v\left\langle e, \frac{\partial e}{\partial \xi}\right\rangle_{\Omega}-\left\langle e, Q_{e} e\right\rangle_{\Omega}
$$

The right hand side terms in equation (14) are bounded as (see [8] and [7]):

$$
\left\langle e, D_{e} \frac{\partial^{2} e}{\partial \xi^{2}}\right\rangle_{\Omega} \leq-\delta \lambda_{1}\|e\|_{\Omega}^{2}
$$




$$
\begin{gathered}
\left\langle e, \frac{\partial e}{\partial \xi}\right\rangle_{\Omega}=\langle e, e\rangle_{\Gamma} \geq 0 \\
\left\langle e, Q_{e} e\right\rangle_{\Omega} \geq q\|e\|_{\Omega}^{2}
\end{gathered}
$$

where $\delta$ and $q$ correspond with the lower bounds in (2) and $\lambda_{1}>0$ is the first eigenvalue associated to the equation [15]:

$$
\frac{\partial^{2} \phi_{i}}{\partial \xi^{2}}=-\lambda_{i} \phi_{i}
$$

Combining inequalities (14) with (15) we then get:

$$
\frac{d \mathcal{V}}{d t} \leq-\delta \lambda_{1}\|e\|_{\Omega}^{2}-q\|e\|_{\Omega}^{2}
$$

And the result follows by noting that $\mathcal{V}=2\|e\|_{\Omega}^{2}$ and applying Gronwall lemma [16] to equation (17) so that:

$$
\|e\|_{\Omega}^{2} \leq\|e(0)\|_{\Omega}^{2} \exp (-\mu t)
$$

with $\mu=\left(\delta \lambda_{1}+q\right) / 2$

\section{Remark 1}

As it can be concluded from (18) the exponential convergence of the observer will depend on process design parameters such as the reactor size (implicit in $\lambda_{1}$ ), degree of dispersion or mass and energy transfer coefficients.

\section{OBSERVER IMPLEMENTATION}

In order to implement the proposed observation scheme (10) the PDE set needs to be solved on-line and the complete field $x_{m}$ measured. Standard PDE solvers are based on finite differences (FD) or finite element methods (FEM) which essentially consist of spatial discretization schemes that approximate the original PDEs by an usually large set of ordinary differential equations (ODEs). In particular, FEM is based on Galërkin projection of the original PDEs over a set of locally defined basis functions $\left\{\psi_{i}\right\}_{i=1}^{n}$ [11]. When applied to (10) the resulting ODE set becomes of the form:

$$
\begin{gathered}
\dot{Z}_{j}=\mathcal{D} \mathcal{A}^{-1}\left[-\left(D_{j} \mathcal{C}+v \mathcal{Q}+v \mathcal{B E}\right) Z_{j}-Q_{j}\left(Z_{j}^{*}-Z_{j}\right)+\right. \\
\left.+v \mathcal{G}+h_{j}\left(x_{m}\right)\right]
\end{gathered}
$$

where subscript $j$ extends to the dimension of $z$ and $Z_{j} \in$ $\mathbb{R}^{n}$ corresponds with the discrete version of the original distributed fields $z_{j}$. Using the notation introduced in (11) for domain and boundary integrals, the right hand side terms of (19) are computed as:

$$
\begin{gathered}
\mathcal{B E}_{i j}=\left\langle\frac{\partial \psi_{i}}{\partial \xi}, \psi_{j}\right\rangle_{\Omega} \mathcal{C}_{i j}=\left\langle\frac{\partial \psi_{i}}{\partial \xi}, \frac{\partial \psi_{j}}{\partial \xi}\right\rangle_{\Omega} \\
\mathcal{D} \mathcal{A}_{i j}=\left\langle\psi_{i}, \psi_{j}\right\rangle_{\Omega} \mathcal{Q}_{i j}=\left\langle\psi_{i}, \psi_{j}\right\rangle_{\Gamma} \mathcal{G}_{i j}=\left\langle\frac{\partial \psi_{j}}{\partial \xi}\right\rangle_{\Gamma}
\end{gathered}
$$

As pointed out in [4] and [17], approaches based on spatial discretization are usually computationally involved and present a number of disadvantages as some essential controltheoretic properties such as controllability or observability may be lost by the discretization scheme or the degree of refinement. Moreover, complete measurements of the field $x_{m}$ are not always possible due to the limited number of sensors usually available, which calls for efficient field reconstruction schemes from a reduced number of sensors [5] [6]. These questions will be properly addressed next in the framework of reduced order modelling (ROM) of dissipative systems as developed in [9], [6] and [10].

\section{A. Reduced order model representation}

In constructing a reduced order dynamic representation for (10) we make use of the dissipative nature of diffusionconvection systems and expand $Z_{j}(t, \xi)$ (the discrete version of the field $z_{j}$ ) as a complete series of orthonormal globally defined basis functions $\left\{\phi_{i}(\xi)\right\}_{i=1}^{\infty}$ so that:

$$
Z_{j}(t, \xi)=\sum_{i=1}^{\infty} c_{i}^{j}(t) \phi_{i}(\xi)
$$

where each $\phi_{i}$ is computed by solving the following eigenvalue problem:

$$
\int_{\Omega} R\left(\xi, \xi^{\prime}\right) \phi_{i}\left(\xi^{\prime}\right) d \xi^{\prime}=\lambda_{i} \phi_{i}(\xi)
$$

with $\lambda_{i}$ being the eigenvalue associated to each eigenfunction $\phi_{i}$. Depending on the nature of the kernel $R$ different sets of basis functions emerge [6], among which the following are considered:

1) Spectral decomposition, where $R$ is the Green function associated with the spatial operator.

2) Proper Orthogonal Decomposition (POD), where $R$ is a two point correlation matrix constructed from empirical data (snapshots).

The ordered structure of the eigenspectrum $\left\{\lambda_{i}(\xi)\right\}_{i=1}^{\infty}$ defines a set of low dimensional subspaces $\left\{\phi_{i}(\xi)\right\}_{i=1}^{k}$ which approximate the original field, thus guiding the selection of such subspace which captures most of the relevant dynamic features of the solution. Once we define the subspace, the field is approximated by a truncated series expansion of the form:

$$
Z_{j}(t, \xi) \cong \sum_{i=1}^{k} c_{i}^{j}(t) \phi_{i}(\xi)=\Phi(\xi) C_{j}(t)
$$

where $\Phi$ and $C_{j}$ collect in matrix and vector form, respectively, the sets $\left\{\phi_{i}(\xi)\right\}_{i=1}^{k}$ and $\left\{c_{i}^{j}(t)\right\}_{i=1}^{k}$. The low dimensional dynamic representation is then constructed by projecting (10) onto the basis $\left\{\phi_{i}(\xi)\right\}_{i=1}^{k}$ to obtain the following reduced set of ODEs:

$$
\begin{aligned}
\dot{\widehat{C}}_{j}= & -\left(D_{j} \Lambda-v\left\langle\Phi, \frac{\partial \Phi}{\partial \xi}\right\rangle_{\Omega}-Q_{j}\right) \widehat{C}_{j}+ \\
& +\left\langle\Phi, h_{j}\left(x_{m}\right)\right\rangle_{\Omega}+Q_{j}\left\langle\Phi, Z_{j}^{*}\right\rangle_{\Omega}
\end{aligned}
$$

where $\Lambda$ is a diagonal matrix with the absolute value of the eigenvalues of equation (22). The FEM structure representation (19)-(20) is used to compute the right hand side terms of equation (24) by approximating spatial differential and integral operators by their FEM algebraic counterparts [17]. Given a pair of arbitrary functions $f$ and $g$ continuous in space, and $\mathcal{L}(\cdot)$ being a spatial continuous operator, such as a gradient or laplacian, the correspondences are as follows:

$$
\mathcal{L}(g) \rightarrow \mathcal{D} \mathcal{A}^{-1} P G \quad\langle f, l\rangle_{\Omega} \rightarrow F^{T} \mathcal{D} \mathcal{A} L
$$


where $F, G$ and $L$ are the discrete (FEM) version of functions $f, g$ and $l=\mathcal{L}(g)$, respectively, and $P$ represents either $\mathcal{B E}$ (gradient) or $\mathcal{C}$ (laplacian) in (20). With these correspondences, the reduced order version of the observer becomes:

$$
\begin{gathered}
\dot{\widehat{C}}_{j}=\left(D_{j} \Lambda-v \Phi^{T} \mathcal{B} \mathcal{E} \Phi-Q_{j}\right) \widehat{C}_{j} \\
+\Phi^{T} \mathcal{D} \mathcal{A} h_{j}\left(x_{m}\right)+Q_{j} \Phi^{T} \mathcal{D} \mathcal{A} Z_{j}^{*}
\end{gathered}
$$

\section{B. Optimal field reconstruction from a limited set of mea- surements}

Observer (10) implemented either in the FEM framework or as a reduced order observer (25) requires on-line measurements of the complete field $x_{m}$ which are not always available. In this section, we extend the results developed in [6] to set up the location of sensors for continuous distributed bioprocesses that will allow us to reconstruct in an optimal way the complete field from a limited number of measurements. To that purpose, and in the same way as in (23), let us denote each element of the measurement vector field $x_{m}$ as $u$, and expand it in terms of the basis function set $\left\{\phi_{i}(\xi)\right\}_{i=1}^{k}$, so that:

$$
u \cong\left[\phi_{1}, \ldots, \phi_{k}\right] C_{m} \equiv \Phi C_{m}
$$

Before proceeding with the reconstruction problem, let us first note that basis functions are orthonormal with respect to the spatial integration operator $\mathcal{D} \mathcal{A}$ so that $\Phi^{T} \mathcal{D} \mathcal{A} \Phi=I$. In the same way, by partitioning the spatial domain into $N$ disjoint subdomains, the above orthonormality condition can be re-written as:

$$
\sum_{i=1}^{N} \Phi_{i}^{T} \mathcal{D} \mathcal{A}_{i} \Phi_{i}=I
$$

where $\Phi_{i} \in \mathbb{R}^{n_{i} \times k}, \mathcal{D} \mathcal{A}_{i} \in \mathbb{R}^{n_{i} \times n_{i}}$ and $n_{i}$ are defined for each subdomain. In particular, $\mathcal{D} \mathcal{A}_{i}$ corresponds with the spatial integration operator associated to subdomain $i$ which, as $\mathcal{D} \mathcal{A}$, is invertible and thus accepts the following factorization:

$$
\mathcal{D} \mathcal{A}_{i}=W_{i} \Lambda_{i} W i^{T}
$$

with $\Lambda_{i}$ being diagonal and $W_{i}$ unitary matrices. The reconstruction problem can now be stated as follows:

For a given set of subdomains $\mathcal{S}$ where sensors are located, find the vector $C_{m}$ in (26) which minimizes the distance between the set of measurements $u_{\mathcal{S}}$ and the estimates $\widehat{u}_{\mathcal{S}}$. Its solution, once cast into a least squares minimization problem [6], takes the form:

$$
\widehat{C}=\Pi^{-1} \sum_{i \in \mathcal{S}} \Phi_{i}^{T} \mathcal{D} \mathcal{A}_{i} u_{i} \quad \Pi=\sum_{i \in \mathcal{S}} \mathcal{Z}_{i}^{T} \mathcal{Z}_{i}
$$

where matrices $\mathcal{Z}_{i}$ are computed as:

$$
\mathcal{Z}_{i}=\Lambda_{i}^{1 / 2} W i^{T} \Phi_{i} \quad \text { with } \quad \Phi_{i}^{T} \mathcal{D} \mathcal{A}_{i} \Phi_{i}=\mathcal{Z}_{i}^{T} \mathcal{Z}_{i}
$$

Equation (29) suggests a criterion to place sensors based on the degree of conditioning of matrix $\Pi$. In this way, sensors will be placed over those subdomains $\mathcal{S}$ which maximize the minimum eigenvalue of $\Pi$. Formally this problem is stated as:

$$
\max _{\mathcal{S}} \min _{j_{k}} \lambda_{j}\left(\sum_{i \in \mathcal{S}} \mathcal{Z}_{i}^{T} \mathcal{Z}_{i}\right)
$$

and solved by means of the guided search algorithm developed by [6].

\section{GLUCONIC ACID DYNAMIC MODEL}

\section{A. Reaction mechanism}

Experiments carried out in a batch fermenter (see [13] for details) indicate that the growth of the microorganisms is a diauxic process with logistic and linear phase. During the logistic phase glucose $(\mathrm{G})$ is rapidly consumed by the microorganisms $(\mathrm{X})$ to produce Gluconic Acid (GA), being the glucoholactone (GOT) the intermediate compound, as a result of the action of Glucose Oxidase (GOD). Microorganisms also produce catalasa (CAT) that breaks the hydrogen peroxide $\left(\mathrm{H}_{2} \mathrm{O}_{2}\right)$. This suggests the following reaction mechanism [12]:

$$
\begin{gathered}
G+X \longrightarrow X \\
G+\mathrm{O}_{2} \stackrel{\text { GOD }}{\longrightarrow} \mathrm{GOT}+\mathrm{H}_{2} \mathrm{O}_{2} \\
\mathrm{GOT}+\mathrm{H}_{2} \mathrm{O} \longrightarrow G A \\
\mathrm{H}_{2} \mathrm{O}_{2} \stackrel{\text { CAT }}{\longrightarrow} \mathrm{H}_{2} \mathrm{O}+\frac{1}{2} \mathrm{O}_{2}
\end{gathered}
$$

Once the glucose has been exhausted, the linear phase starts and the microorganisms begin consuming GA with a linear kinetics. In a continuous process, the logistic phase will determine the dynamic model to be obtained from the following set of equations [13]:

$$
\begin{gathered}
R_{X}=\mu^{X} X \frac{k_{1}^{X}-X}{k_{1}^{X}} ; \quad \mu^{X}=\frac{\mu_{\max }^{X} G}{k_{2}^{X}+G} \\
C A T=b G O D \\
R_{G A}=\mu^{G A} G A \frac{k_{1}^{G A}-G A}{k_{1}^{G A}} ; \quad \mu^{G A}=\frac{\mu_{\max }^{G A} G}{k_{2}^{G A}+G} \\
R_{H_{2} O_{2}}=R_{G A}-C A T \frac{V_{\max } H_{2} O_{2}}{k_{m}+H_{2} O_{2}} \\
R_{G O D}=\alpha R_{X}+\beta X \\
R_{G O D}^{T}=k_{1} G O D+k_{2} G O D H_{2} O_{2}
\end{gathered}
$$

The fast consumption of $G$ to produce GA, motivates the following simplified mechanism derived from (31) and more suitable for identification and control:

$$
\begin{gathered}
G+X \stackrel{R_{X}}{\longrightarrow} X \\
G+\frac{1}{2} O_{2} \stackrel{R_{G A}}{\longrightarrow} G A
\end{gathered}
$$

where the biomass and gluconic acid reaction rates correspond with (32) and (34), respectively. 
TABLE I

MODEL PARAMETERS

\begin{tabular}{|c||c|}
\hline Design parameter & Symbol Value $\&$ Units \\
\hline Mass dispersion coefficient & $\mathrm{D}=0.01 \mathrm{~m}^{2} \mathrm{~h}^{-1}$ \\
\hline Flow velocity & $\mathrm{v}=0.01 \mathrm{mh}^{-1}$ \\
\hline Mass exchange parameter & $\mathrm{kla}=600 \mathrm{~h}^{-1}$ \\
\hline Dissolved oxygen & $O_{2}^{*}=7.5 \mathrm{e}-3 \mathrm{gl}^{-1}$ \\
\hline Glucose stream & $G_{i n}=150 \mathrm{gl}^{-1}$ \\
\hline Dissolved oxygen stream & $O_{2 i n}=7.5 \mathrm{e}-3 \mathrm{gl}^{-1}$ \\
\hline \hline Biomass reaction & Gluconic acid reaction \\
rate parameters & rate parameters \\
\hline$\mu_{m a x}^{X}=0.219 \mathrm{~h}^{-1}$ & $\mu_{\max }^{G A}=0.312 \mathrm{~h}^{-1}$ \\
\hline$k_{1}^{X}=2.53 \mathrm{gl}^{-1}$ & $k_{1}^{G A}=109 \mathrm{gl}^{-1}$ \\
\hline$k_{2}^{X}=5 \mathrm{gl}^{-1}$ & $k_{2}^{G A}=1 \mathrm{gl}^{-1}$ \\
\hline
\end{tabular}

\section{B. The Distributed Dynamic Model}

The process takes place on a tubular reactor with design parameters and feed streams given in table I. In accordance with the general dynamic structure proposed in (1) and taking into account the simplified mechanism (38), the state vector field for this process becomes $x=\left[\begin{array}{cccc}X & G A & G & O_{2}\end{array}\right]^{T}$. In addition, the yield matrix and reaction kinetics is formally written as:

$$
K=\left[\begin{array}{cc}
1 & 0 \\
0 & 1 \\
-1 & -1 \\
0 & -0.5
\end{array}\right] \quad \varphi(x)=\left[\begin{array}{c}
R_{X} \\
R_{G A}
\end{array}\right]
$$

\section{OBSERVER VALIDATION}

The observation scheme for continuous gluconic acid production is designed, as discussed in Sections II and III, to produce estimates of biomass and gluconic acid from a limited number of measurements of oxygen and glucose so that according to (8) and (39) we have:

$$
\begin{array}{r}
x_{e}=\left[\begin{array}{c}
X \\
G A
\end{array}\right] \quad x_{m}=\left[\begin{array}{c}
G \\
O_{2}
\end{array}\right] \\
K_{e}=\left[\begin{array}{ll}
1 & 0 \\
0 & 1
\end{array}\right] \quad K_{m}=\left[\begin{array}{cc}
-1 & -1 \\
0 & 0.5
\end{array}\right]
\end{array}
$$

The rest of matrices are null except the one that includes the oxygen exchange parameter:

$$
Q_{m}=\left[\begin{array}{cc}
0 & 0 \\
0 & k l a
\end{array}\right]
$$

In order to test the field reconstruction and estimation capabilities on a dynamic context, $\pm 5 \%$ and $\pm 10 \%$ perturbations around steady state operation were implemented in both glucose input concentration and the oxygen input.

The appropriate number of sensors and optimal locations for glucose and oxygen concentration measurements in the reactor, were selected as discussed in Section IIIB from a POD basis set computed by solving (22) from a sufficiently rich set of dynamic snapshots. The POD set consisted of three and one elements for glucose and oxygen concentrations, respectively. The optimal location of sensors was obtained by solving the max-min problem (30) with the guided search algorithm developed by [6]. Their placement along the reactor is depicted in Fig. 1.

These measurements were employed to reconstruct the whole field by means of equations (26) and (29). As it can be seen in Fig. 2 for glucose concentration, the reconstruction error remains extremely low in the event of perturbations thus showing good reconstruction properties. The same behavior was observed for the case of oxygen reconstruction.

Gluconic acid and biomass were estimated from the observer structure given by (10a) and (10b) and implemented on the reduced order modelling framework discussed in Section III, equations (23) and (25). The selected basis function set for this observer corresponds with the spectral eigenfunctions associated to the Laplacian operator.

A comparison, in terms of modes $C$, between a FEM and a reduced order model implementation of the observer (10a) and (10b) is presented in Fig. 3. As shown in the figure, the number of active modes capturing the slow (relevant) dynamics is small (only three modes involved) which translates into a low dimensional dynamic system described by three ODEs for the gluconic acid field to be estimated. This is in contrast with the FEM implementation which requires a much larger set of ODEs for each field (31 in our case). In addition, the comparison highlights the excellent agreement between the ROM and the FEM implementations.

The evolution of the gluconic acid and biomass concentration errors in time and along the reactor are presented in Fig. 4 and Fig. 5, respectively, illustrating the exponentialtype error convergence property discussed in Proposition 1 and demonstrating the good estimation capabilities of the reduced order observation scheme. Furthermore, and as it can be seen by comparing Fig. 5 and Fig. 6, the ability of the reduced order observer to produce reliable estimates is practically the same as the one directly based on FEM but requiring a considerably smaller computational effort.

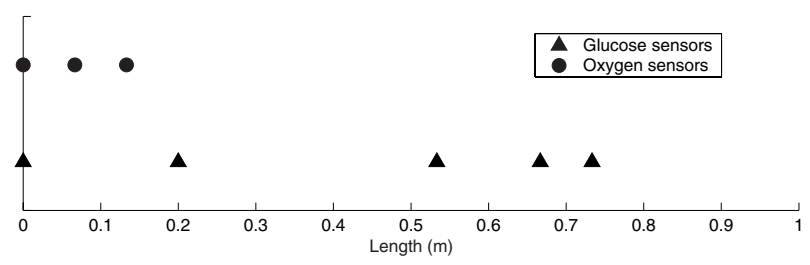

Fig. 1. Optimal placement of sensors for glucose and oxygen in the reactor

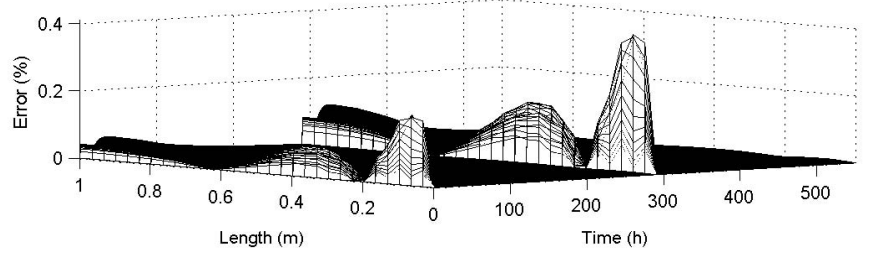

Fig. 2. Evolution of the relative error (\%) during the reconstruction of glucose concentration from partial measurements 

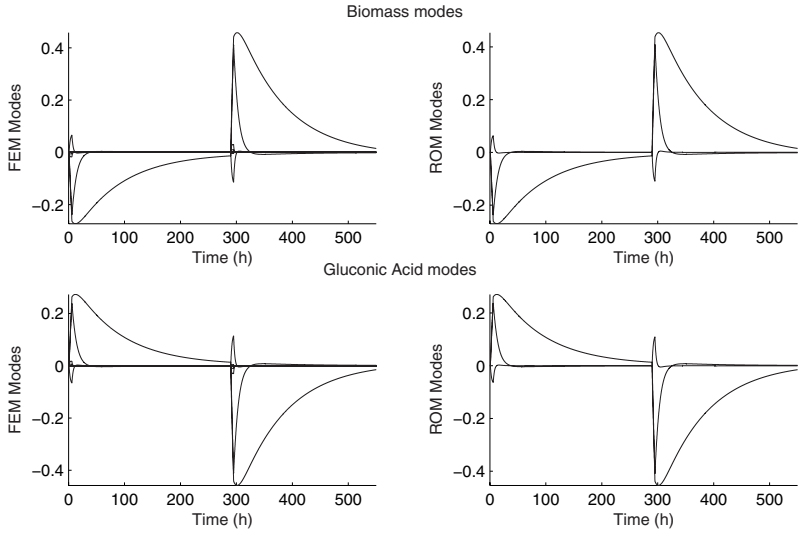

Fig. 3. Evolution of the observer modes $C(t)$ for biomass and gluconic acid corresponding to a FEM and a ROM implementation

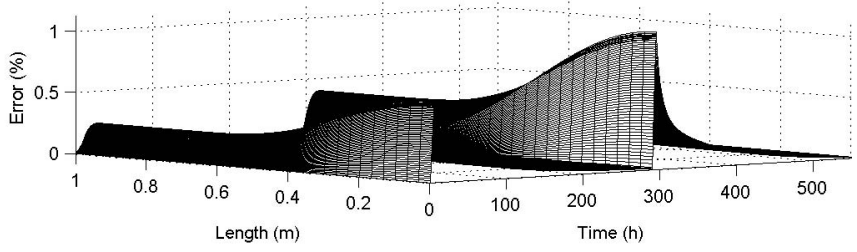

Fig. 4. Evolution of the ROM observer error in the estimation of gluconic acid from partial measurements

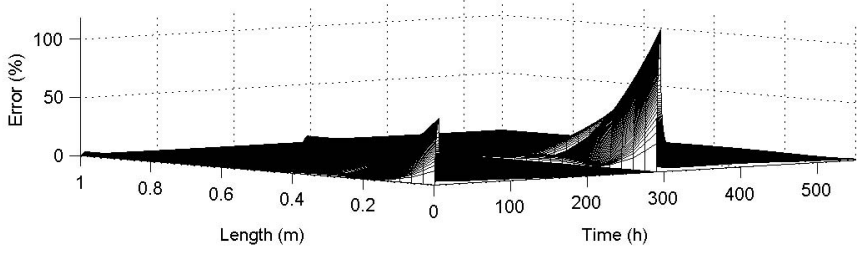

Fig. 5. Evolution of the ROM observer error in the estimation of biomass from partial measurements

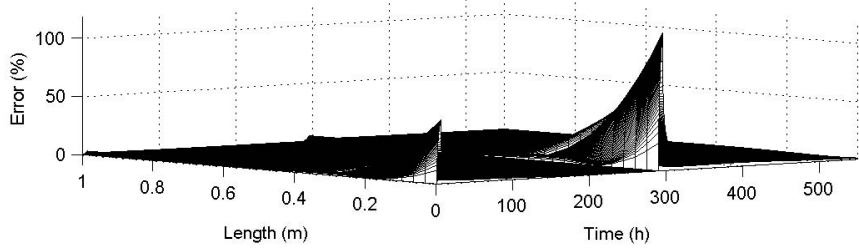

Fig. 6. Evolution of the FEM observer error in the estimation of biomass from partial measurements

\section{CONCLUSIONS}

In this work, a novel systematic observer design methodology for spatially distributed continuous bioreactors was presented. The approach, which extends further the theory of bioreactor estimation developed by Bastin and Dochain to diffusion-convection processes, takes advantage of its dissipative nature to derive robust and efficient low order dimensional observation schemes.
On the proposed framework, practical aspects of importance in industrial implementation such as the optimal location of a given limited number of sensors can be also easily handled. The application of this methodology has been illustrated on a case study of industrial relevance such as the continuous production of gluconic acid.

\section{ACKNOWLEDGMENTS}

This paper includes results of the joint research project that is supported by the bilateral agreements between Bulgarian academy of sciences and Process Engineering Group IIMCSIC. The authors acknowledge financial support received from the Spanish Government (MCyT Projects PPQ20013643) and Xunta de Galicia (PGIDIT02-PXIC40209PN).

\section{REFERENCES}

[1] G. Bastin and D. Dochain, On-line Estimation and Adaptive Control of Bioreactiors, Elsevier, Amsterdam; 1990.

[2] D. Dochain, N. Tali-Maamar and J.P. Babary, On modelling, monitoring and control of fixed bed bioreactors, Computers chem. Engng, vol 21, 1996, pp 1255-1266.

[3] D. Dochain, State observers for tubular reactors with unknown kinetics, Journal of Process Control, vol 10, 2000, pp 259-268.

[4] P.D. Cristofides, Nonlinear and Robust Control of PDE Systems: Methods and Applications to Transport-Reaction Processes, Birkhäuser, Boston; 2001

[5] A.V. Wouwer, N. Point, S. Porteman and R. Remy, An approach to the selection of optimal sensor locations in distributed parameter systems, Journal of Process Control, vol 10, 2000, pp 291-300

[6] A.A. Alonso, C.E. Frouzakis and I. G. Kevrekidis, Optimal Sensor Placement for State Reconstruction of Distributed Process Systems, AIChE Journal, vol 50, 2004, pp 1438-1452.

[7] A.A. Alonso and B.E. Ydstie, Stabilization of Distributed Process Systems using irreversible thermodinacis, Automatica, vol 37, 2001, pp 1739-1755.

[8] A.A. Alonso, J.R. Banga and I. Sánchez, Passive Control Design for Distributed Process Systems: Theory and Applications, AIChE Journal, vol 46, 2000, pp 1593-1606.

[9] A.A. Alonso, C.V. Fernández and J.R. Banga, Dissipative systems: from physics to robust nonlinear control, Int. J. Robust Nonlinear Control, vol 14, 2004, pp 157-179.

[10] E. Balsa-Canto, A.A. Alonso and J.R. Banga, Reduced-Order Models for Nonlinear Distributed Process Systems and Their Application in Dynamic Optimization, Ind. Eng. Chem. Res., vol 43, 2004, pp $3353-$ 3363.

[11] J.N. Reddy, An introduction to the Finite Element Method, McGrawHill, United States; 1993.

[12] V.R. Rai and A. Constantinides, Mathematical Modeling and Optimization of the Gluconic Acid Fermentation, AIChE symp. Ser., vol 69, 1973, pp 114-122.

[13] J. Mirón, M.P. González, L. Pastrana and M.A. Murado, Diauxic production of glucose oxidase by Aspergillus niger in submerged culture. A dynamic model, Enzime and Microbial Technology, vol 31 2002, pp 615-620.

[14] I. Famili and B. O. Palsson, The Convex Basis of the Left Null Space of the Stoichiometric Matrix Leads to the Definition of Metabolically Meaningful Pools, Biophysical Journal, vol 85, 2002, pp 16-26.

[15] J. Smoller, Shock Waves and Reaction-Diffusion Equations, Springer, New York; 1983.

[16] H.K. Khalil, Nonlinear systems, Prentice-Hall, Englewood Cliffs; 1996

[17] C. Vilas, M.R. García, M.R. Fernández, E. Balsa-Canto, J.R. Banga and A.A. Alonso, "On Systematic Model Reduction Techniques for Dynamic Optimization and Robust Control of Distributed Process Systems", ESCAPE 14, Lisbon, 2004. 